\section{SOME POINTS IN THE DIAGNOSIS OF GALL-STONES.*}

\section{JAMES B. HERRICK, M.D.} CHICAGo.

Repeated attacks of colic with the pain in the right hypochondrium, accompanied by nausea and vomiting and followed in a few hours by a slight jaundice, leave little doubt as to the existence of gall-stones. In the maiority of cases it is on a history of such attacks with the finding of the calculi in the feces that the diagnosis is made.

But the stones are not always so frank in making known their presence. They manifest themselves by irregular symptoms, they simulate other diseases, and other diseases simulate them, so that it is true, as HoppeSeyler says, that if the uncomplicated, regular cholelithiasis is difficult to recognize, much more difficult is it to recognize the irregular and complicated form of the disease. ${ }^{1}$ It is to some of the symptoms most likely to be misinterpreted and to some diseases liable wrongly to be regarded as gall-stones that I wish to direct attention.

Pain.-It is well known that the pain of gall-stone colic may not be limited to the right hypochondrium. A favorite spot for its reflection-by way of the phrenic nerve and the cervical plexus - is to the right shoulder and close to the angle of the right scapula. It may be referred to the back and make one think of renal colic, may even shoot downward and toward the genitalia, or be located to the left of the median line, in which condition Mayo Robson has found pyloric adhesions. ${ }^{2}$ But che point of reference of the pain that causes many mislakes in diagnosis is one which is frequently complained $\because f$ by patients and frequently described in articles on the subject, viz., the epigastrium. Recurrent attacks of nansea and vomiting, because they come on a few hours after meals and because there is complaint of pain in the region of the stomach, are repeatedly called gastric neuralgia or acute gastric indigestion, and a slight subsequent jaundice is overlooked or is passed by as a simple accompanying catarrhal jaundice. Attacks of "gastric neuralgia" recurring at irregular intervals, the attacks coming on several hours after meals and especially at night should arouse the suspicion of gall-stones and cause the sclera and urine to be examined for evidence of icterus and the stools for acholia and stones.

Icterus.-Not counting the cases of hepatic colic in which jaundice is overlooked because it is slight, or is not sought for, there are many in which no evidence of icterus is to be discovered, even by the most searching scrutiny of the skin, sclera and urine, and at no time are the feces pale because of absent bile-pigment. These cases often prove confusing and errors are made because of the lack of icterus, which has rightly been considered one of the most important diagnostic aids but which is so frequently absent that it can not be regarded as in any sense pathognomonic or even as an essential factor in diagnosis. In reality, aside from the finding of stones in the feces there is no symptom or sign that is pathognomonic. The diagnosis is one of probability and is made by the-symptom-complex.

Jaundice may be lacking after colic because the stone, even though in motion, may drop back into the gallbladder, or from near the narrow choledochus opening back into the wider portion of the common duct and

\footnotetext{
* Read at the meeting of the North Central Ohio Medical Socie ty, Mansfield, Ohlo, Sept. 28, 1900.
}

not enough swelling result to produce the obstructive jaundice. Or small or smooth stones may pass quickly and leave so little local damage and swelling that there is no stagnation of bile. Wolff saw icterus in only onehalf of his cases, though, in all, gall-stones were found in the stools. And Fürbringer ${ }^{4}$ in three-quarters of his cases failed to find jaundice. Angular or faceted stones may even lodge in the common duct and, because of their shape, leave enough space for the escape of bile, so that the feces are still bile-tinged and no discoloration of the skin, mucous membrane or urine occurs.

These are conditions in which stones moving from the gall-bladder through the cystic into the common duct, and perhaps escaping therefrom, may still leave no trace of their existence in the biliary tint of the skin or urine. But there is a large class of cases of biliary colic in which infiammation plays an important, if not the sole, part. Riedel in particular has called attention to them. These are cases of stone in the gallbladder where an acute inflammation or the lighting up of a dormant inflammation occurs, the condition being one therefore of cholecystitis and cholangitis, at least a portion of cystic duct being inflamed. The bacteria producing it may have entered from the intestine and primarily have caused the formation of the stone through the inflammation excited, or they may have entered later and have lain quiet so long as conditions were not particularly favorable for their development, or, perhaps, so long as there was good drainage through the cystic duct. But for some reason, at a given time, be it increase in numbers or virulence of the microbes, or movement of the stone to the mouth of the bladderduct, there is an acute inflammation of the gall-bladder swelling of its mucosa and that of the cystic duct; the outlet is therefore occluded. Pain, nausea and vomiting are present, and in addition there is tenderness over the gall-bladder; this in many instances may be made out as distinctly enlarged, being distended with the retained bile and inflammatory mucoserous, or mucopurulent exudate. The temperature, if carefully taken, will be found slightly elevated, from one to two or three degrees, above the normal, and may so remain for a few days, as may the local tenderness and swelling. Some of these cases are complicated by a slight, local peritonitis, repeated attacks resulting in adhesions and thickenings about the gall-bladder. The important fact, the misleading fact as it would appear from experience, is that there is no jaundice. The probable diagnosis must be made without its presence, upon the history of previous attacks, the location of the pain, especially over the region of the gall-bladder, its radiation, tenderness over the region of the gall-bladder, the enlargement of the viscus and the slight temperature. No definite statements can as yet be made as to the value of the direction of radiation of the pain as an aid toward locating the stone. It has seemed in some cases, and also from observations made while probing biliary fistulæ, as though pain referred to the right scapular region might mean stone in the cystic duct. The importance of early recognition of gall-bladder stones before chronic inflammatory or ulcerative changes in the bladder and its immediate neighborhood have taken place, or the stone has, perhaps, become lodged in the common duct is self-evident, rendering surgical interference possible at a time when the operation is easier and safer and brings about great results in the way of avoiding unfortunate complications and sequelæ of calculi. 
Fever.-Since the bacterial era fever in connection with gall-stones has been generally looked on as an evidence of microbic invasion of the biliary tract or of neighboring organs, e. g., the liver, that have been secondarily involved. I wish to make brief reference merely to the fever commonly known, after Charcot, as the hepatic intermittent fever. This is associated with stone or stones in the common duct. Pus or bacteria that can be cultivated, and naked-eye changes in the mucosa are frequently absent. The exact nature of the fever, whether microbic, toxic or both, is still uncertain. It has been compared to urethral fever. In many cases there is jaundice that persists for months or years. At irregular periods chill, fever. sweating, pain in the right hypochondrium, often vomiting, occur, and the jaundice for a time deepens. The freedom with which some of the stones change their position in the duct and the manner in which they intermittently occlude the duodenal opening of the common duct produeing jaundice, or of the cystic duct leading to atrophy of the gall-bladder, has been clearly shown by Fenger, in his article on "Stones in the Common Duct and their Surgical Treatment, with Remarks on the Ball-Valve Action of Floating Choledochus Stone."

If in every such case of intermittent hepatic fever there is this periodic closure of the exit of the choledochus, the paroxysm would seemingly be due to retention and absorption of some yet unrecognized toxic material. Osler; ${ }^{6}$ who early called attention to this from of gall-stone manifestation, lays, as it seems to me, a little too much stress on the permanence of the jaundice and its invariable appearance after the attack of chill, fever and colic. If in some cases there is an incomplete occlusion, the obstruction may be so slight as to lead to no persistent jaundice. At the time of the paroxysm there is more complete obstruction, but it may be of such short duration that stagnation of the bile and absorption by the lymphatics does not take place to an appreciable extent, and no jaundice appears. That this absence of icterus for long periods, and even after attacks of chill, fever and colic, is not only theoretically the fact but is actually so, is proved by histories of some of Fenger's cases. And I have in two instances seen no jaundice appear after chill, fever, sweat and pain, though, because gall-stones were suspected, the patients, who were under observation in a hospital, were most carefully watched for evidences of the slightest trace of icterus, which ultimately appeared, but was intermittent. And right here I would emphasize the importance, in doubtful cases, of putting the patient in the hospital and keeping him under observation for days or weeks, if necessary, so that the course of the fever, the evidence of icterus in the skin, stools or urine may be properly judged. In both the cases above referred to, it was in this way and this alone that a correct diagnosis was made, as shown by the removal of solitary choledochus stones. The diagnosis in the cases with permanent jaundice or jaundice plainly perceptible after each attack is comparatively easy. The same chill, fever and sweat have been observed in obstruction from pressure, e. g., from carcinoma, hence caution has to be observed in making too positive an ante-operation diagnosis.

Association with Carcinoma.-In the adult and aged particularly, the association of gall-stones and carcinoma is so common as to be regarded as more than accidental. While the question as to which is the primary trouble is not positively settled, the preponderance of evidence is in favor of the view that the gall-stone is in most cases the primary disease, the carcinoma arising in the gall-bladder or bile-duct at the point of irritation by the stone. The possibility of carcinoma being found along with the calculus should make one circumspect as to his diagnosis and prognosis, particularly when with advanced age there is an unusually rapid loss of weight and strength, with anemia, and increasing size of the liver that is in these cases so often the seat of secondary growths by direct extension or metastasis. Palpable and perhaps nodular masses in the region of the gallbladder, when associated with cachexia, would arouse suspicion of a neoplasm. Yet inflammatory exudates and thickening of tissue will readily deceive. In fact, even with the abdomen opened, the surgeon is often in doubt as to whether the hard and thickened tissue in the neighborhood of the stone is inflammatory or neollastic.

Riedel's Tongue-Shaped Process.-A projection of the lower, i. e., anterior border of the liver as a tongueshaped process has been described by Riedel as found in miny cases of gall-bladder stone where there is pericystic thickening. This tongue-like projection is ofieriest seen in women with the corset-liver and gallstone. In its physical properties it is not unlike a tumor of the gall-bladder, the right kidney or the large intestine. If its possible existence is remembered it can frequently be recognized by its shape, its edge, its location, its respiratory mobility, its situation above and in front of colon, as well as by the positive signs and symptoms in the previous history pointing to gall-stones and the absence of symptoms indicating renal or intestinal new-growths. Extremely confusing is the condition in which Riedel's process is crossed by the adherent intestine. The projection is then separated from the bulk of the liver by an area of tympany, especially noticeable with the colon distended with air, and it is not to be wondered at that the process is often regarded as a kidney or as a new growth of the intestine or omentum. Eichhorst, as well as others, speaks particularly of the difficulty of diagnosis in such cases. One such I have seen trip up one of the acutest diagnosticians of Europe.

Relation of Typhoid Fever to Gall-Stone.-The relation between typhoid fever and cholelithiasis is in some cases so close that a history of typhoid a few months or years before adds a probability to a diagnosis of gallstones. The researches of Chiari ${ }^{7}$ and others show the great frequency with which typhoid bacilli are found in the gall-bladder and the bile of patients dying of typhoid, there being in some cases naked-eye evidence of cholecystitis. Recent observations have confirmed these statements, and have shown bacilli in bile many months after the subsidence of the fever. The changes they produce in the mucous membrane of the gallbladder are those favoring the shedding of the epithelium and the increase in the amount of cholesterin, i. e., Naunyn's requirements for the production of calculi are met. Typhoid bacilli have been found in the nuclei of stones; stones have shown themselves by symptoms soon after attacks of typhoid, and autopsies on patients dying several months or a few years after typhoid fever have, in not a few instances, shown gall-stones with typhoid bacilli in the gall-bladder. There seems little reason to doubt that gall-stone formation is favored by the presence of typhoid germs in the gall-bladder, and that they are frequently present in that situation during typhoid. The value, therefore, of inquiry into the history for typhoid is clear.

Conditions that Simulate Gall-Stones.-Thus far I have spoken only of points concerning diagnosis where 
gall-stones are actually present. 1 wish now to call attention to a few conditions that may simulate gallstones and thus lead to error in diagnosis. Ulcer of the stomach, neuralgia of the stomach, renal, intestinal and lead colic are all so freely discussed in text-book articles, and so quickly occur to the mind of one examining a patient with suspected gall-stones, that I will not refer to them further, but take up briefly a few diseases that I have found confusing to myself and others. Theoretically, some of them ought not to confuse. But practice and theory are here, as in many other situations, not always in harmony. Some of these conditions if once thought of will be readily recognized. But one of our great troubles in diagnosis is that our attention is easily attracted by some striking feature of a case toward some particular disease of which it may be a prominent symptom. We are apt to forget that this same symptom may be a minor or exceptional manifestation of some other disease that will be overlooked unless it accidentally comes to our mind, or unless it thrusts itself forward by other symptoms revealed during a thorough routine examination.

To illustrate this point, let me cite the case of a man whom I saw in my ward at the Cook County Hospital, suffering severe pain which he referred chiefly to the epigastrium. In response to my inquiry as to what was the cause of the pain, he replied that it was gall. stones, that he had been operated on for them severa] months before, had had relief for a few months, but now the same periodic pains had returned and he was sure some stones had been overlooked or others had formed. He had the operation-scar to confirm his statements. My first thought was that the man was right, for stones can be readily overlooked at an operation and recurrences are by no means unknown. ${ }^{8}$ But the points to which the pain radiated, into the arms and thighs, made me examine more closely, and I found the ear-marks of tabes-loss of knee-jerk, Romberg's symptom, Argyll-Robertson pupil, etc., and his epigastric pains were but the gastric erises of that disease. I have no reason to question the statement made by the surgeon to the patient, that he removed gallstones but I narrowly escaped wrongly consigning the patient to the surgical ward and to an unnecessary second operation.

Angina Pectoris.-I have once or twice been puzzled to differentiate between gall-stones and angina. The pain of gall-stones may, as has been said, be referred to the left side and may even appear in the breast. and anginal pain may involve the epigastrium. In general, however, the thickened condition of the arteries, the enlargement of the heart, the distinct precordial location of the pain with its radiation to the neck and left arm, the violent, grasping, vise-like nature of the pain with the sense of impending death, the absence of later jaundice, ete., make a diagnosis a simple matter.

Senile Pneumonia.-The pain of pleurisy, especially when it involves the lower right side and the diaphragm, is often referred to the abdomen. With pneumonia there may be vomiting, and not infrequently icterus, the latter perhaps from an accompanying catarrhal ja'ndice. A patient, therefore, with acute epigastric and right hypochondriac pain, nausea and vomiting, chill and fever and subsequent jaundice, resembles in many respects a case of gall-stones. This was first impressed on me by the case of a physician of about 60 years, who called me to see him after he had been ill and treating himself for two days. He told me he thought he had gall-stones and cholecystitis, for he had sharp pain in the region of the gall-bladder, with fever and jaundice. 'The condition was, however, one of right lower lobe pneumonia of the senile type, with comparatively slight cough and expectoration.

Localized Peritonitis.-Acute peritonitis or exacerbation of a chronic peritonitis may, if in the region of the gall-bladder, make one think seriously of gallstones. A man of about 23, who a few years before had passed through an attack of typhoid fever, had been annoyed for ten months with what at first he regarded as indigestion. At irregular intervals, on four or five occasions, he had suffered from poor appetite for a day or two, coated tongue, then severe pain in the epigastrium and right hypochondrium, vomiting and fever. These attacks had gradually subsided, leaving him rather weak, with some soreness just below the right costal arch. His last attack had been quite severe and his physician thought it was due to gall-stones. Jaundice had been doubtful. The history led me to suspect gall-stones with cholecystitis and extension of inflammation to the neighboring peritoneum, for there was in the neighborhood of the gall-bladder, after the acute symptoms had subsided, a resistance and tenderness, though for days no temperature. This diagnosis was concurred in by another physician and by the surgeon who operated. We found an extensive tuberculous peritonitis, but no gall-stones. Recovery from the operation-a simple opening and loosening of adhesionswas followed in the course of several weeks by a great improvement in local and constitutional symptoms and a gain of twenty-five pounds in weight. The patient is still living and reports himself well over a year from the time of operation. Appendicitis, intestinal obstruction, hemorrhagic pancreatitis may resemble gall-stones with inflammation of the gall-bladder or neighboring peritoneum. Of the difficulty of differentiating between gall-stones and intestinal obstruction a case recorded by Fenger $^{2}$ is a good example where an operation done for suspected intestinal obstruction revealed no obstruction, but a distended gall-bladder, yielding on opening, a largo number of ealculi, the symptoms of ileus disippeariug after the removal of the stones.

Hernia of the linea alba. Dietl's crisis in floating kidney, hysteria, malaria with neuralgic pain and jaundice, might be mentioned as conditions occasionally simulating gall-stones, and I must call attention to the not infrequent association of cholelithiasis and other diseases. I have already mentioned typhoid fever and carcinoma of the gall-bladder. Diabetes, gout, obesity and renal calculi are many times found with gall-stones; and, accidentally, any disease may occur in a patient who has gall-stones, as in the case of tabes I have cited.

I have aimed in this rather cursory manner to refer only to a few points, especially those I have found puzzling to myself or others, and have made no effort to go into the details of diagnosis of gall-stones with the numerous possible complications and sequelæ.

BIBLIOGRAPHY

1. Die Krankheiten der Leber. Quincke and Hoppe-Seyler in Nothnagel's System.

2. Allbutt's System, v, p. 241.

3. Quoted by Hoppe-Seyler, p. 220, loc. cit. 1896

Fenger: Am. Jour. of the Med. Sci., February and March,

5. Osler: On Fever of Hepatic Origin, etc.; Johns Hopkins Hospital Reports, i1, 1890. Also, On the Ball-Valve Gall-Stone in the Common Duct; The Lancet, 1897, p. 1319.

6. Zeitschrift f. Heilkunde, Bd. xv, 1894

7. See Hermann: Ueber Recidive nach Gallstelnoperationen Mitteilungen aus den Grenzgebieten der Medizin und Chirurgie, Bd. $\checkmark$, Hft. 3 ; also, Kehr-Halberstadt, Münch. Med. Woch., May 22, 1900. 8. Fenger: Chicago Medical Recorder, April, 1898, p. 310. 